Pacific Journal of Mathematic 


\title{
CATEGORY THEORY APPLIED TO PONTRYAGIN DUALITY
}

\author{
DAVID W. ROEDER
}

A proof of the Pontryagin duality theorem for locally compact abelian (LCA) groups is given, using category-theoretical ideas and homological methods. The proof is guided by the structure within the category of LCA groups and does not use any deep results except for the Peter-Weyl theorem. The duality is first established for the subcategory of elementary LCA groups (those isomorphic with $T^{i} \oplus Z^{j} \oplus R^{k} \oplus F$, where $T$ is the circle group, $Z$ the integers, $R$ the real numbers, and $F$ a finite abelian group), and through the study of exact sequences, direct limits and projective limits the duality is expanded to larger subcategories until the full duality theorem is reached.

Introduction. In this note we present a fairly economical proof of the Pontryagin duality theorem for locally compact abelian (LCA) groups, using category-theoretic ideas and homological methods. This theorem was first proved in a series of papers by Pontryagin and van Kampen, culminating in van Kampen's paper [5], with methods due primarily to Pontryagin. In [10, pp. 102-109], Weil introduced the simplifying notion of compactly generated group and explored the functorial nature of the situation by examining adjoint homomorphisms and projective limits. Proofs along the lines of Pontryagin-van Kampen-Weil appear in the books by Pontryagin [7, pp. 235-279] and Hewitt and Ross [2, pp. 376-380]. A different proof based on abstract Fourier analysis was given by Cartan and Godemont [1]; similar methods are also used by Rudin [9, pp. 27-29] and Heyer [3, pp. 148-161]. Negrepontis [6, pp. 239-252] presented the theorem in light of category theory, but for the most part used different methods and more structure theory than is used here.

The proof we present is based on ideas used previously by Hofmann [4, pp. 109-117] and the author [8]. It rests neither on the structure theorem for compactly generated LCA groups (see [2], [6], [7]) nor on the structure of the $L^{1}$-algebra of $G$ (see [6] and [9]). The only deep result we require is the classical Peter-Weyl theorem, applied in our case to compact abelian groups, of course.

Definitions and preliminaries. $L$ is the category of LCA groups, with continuous homomorphisms as morphisms. Groups will be written additively. Pointwise addition of functions makes each $\operatorname{Hom}(G, H)$ an abelian group and $L$ an additive category. $R$ denotes the additive real numbers with the usual topology, $Z$ is the subgroup of $R$ con- 
sisting of the integers with the induced (discrete) topology, $T$ is the compact quotient group $R / Z$, and $Z_{n}$ is $Z / n Z$. If $G \in L$, then the character group of $G$ is $\chi(G)=G^{*}=\operatorname{Hom}(G, T)$, taken with the compact-open topology, which makes $G^{*}$ an LCA group. For $f \epsilon$ $\operatorname{Hom}(G, H)$, the adjoint homomorphism $\chi(f)=f^{*} \in \operatorname{Hom}\left(H^{*}, G^{*}\right)$ is defined by $f^{*}(\alpha)=\alpha \circ f$ for $\alpha \in H^{*}$. Thus $\chi$ is a contravariant functor from $L$ to $L$. There is a natural transformation $\rho$ from the identity functor $\eta$ in $L$ to the covariant functor $\chi^{2}=\chi \circ \chi$; we specify $\rho_{G}: G \rightarrow$ $G^{* *}$ by $\left[\rho_{G}(x)\right](\alpha)=\alpha(x)$ for $x \in G, \alpha \in G^{*}$. The Pontryagin duality theorem is now the statement that $\rho$ is a natural isomorphism; i.e., that each $\rho_{G}$ is an isomorphism between $G$ and its second character group $G^{* *}$. The method of proof will be to show that $\rho$ restricted to a suitable subcategory is an isomorphism, and then enlarge the subcategory in successive steps until we have the theorem proved. We indicate this schematically in the following diagram, where each name stands for the full subcategory of $L$ with the named property, and the labels on connecting lines indicate the methods used to extend the duality to the larger category.

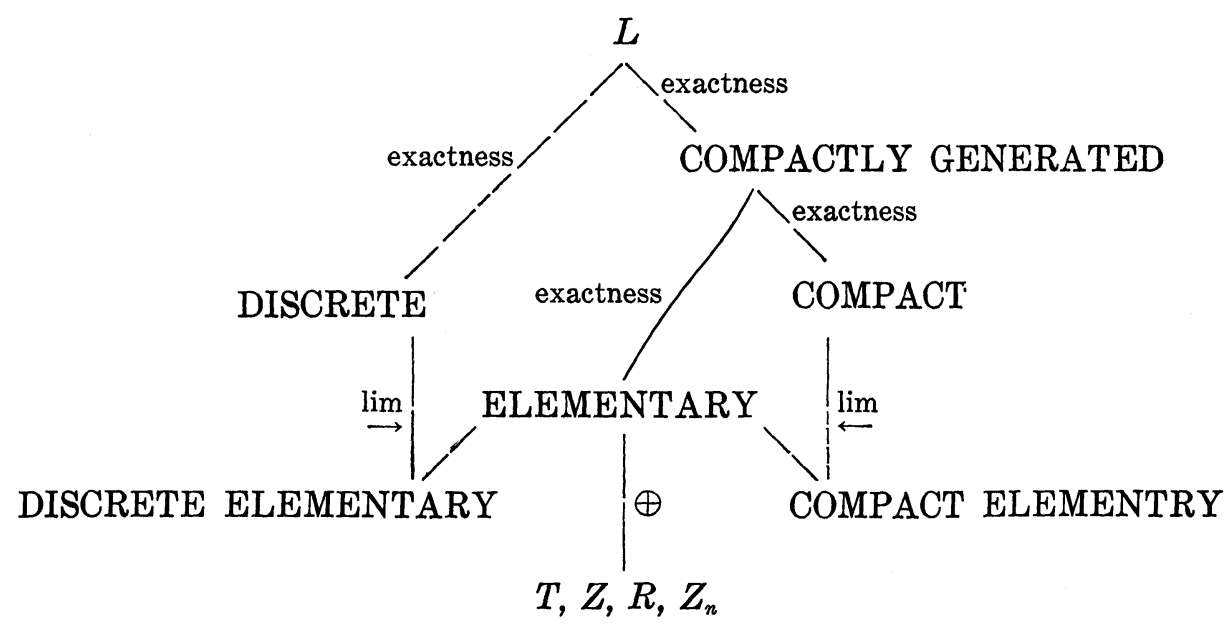

First, $T \simeq Z^{*}$ with $x \in T$ corresponding to the character $n \mapsto n x$ of $Z$. Conversely, $Z \simeq T^{*}$ with $n \in Z$ corresponding to the character $x \mapsto n x$ of $T$. Also $R \simeq R^{*}$ with $x \in R$ corresponding to the character $y \mapsto x y+Z$ of $R$. And $Z_{n} \simeq Z_{n}^{*}$ by corresponding $x \in Z_{n}$ to the character $y \mapsto x y$ of $Z_{n}$. Thus the relation $G \simeq G^{* *}$ holds in a natural way for $G=T, Z, R$, or $Z_{n}$, respectively, and in each case the isomorphism obtained is precisely the map $\rho_{G}$ defined earlier.

It is easy to show that $\chi$ is additive (that is, for $f, g \in \operatorname{Hom}(G, H)$ we have $\chi(f+g)=\chi(f)+\chi(g))$; as a consequence $\chi$ preserves direct sums. (See Lemma 2, p. 162, in [8].) From this we see that Pontryagin duality holds at least for the elementary groups in $L$; 
that is, groups isomorphic to $T^{i} \oplus Z^{j} \oplus R^{k} \oplus F$, where $F$ is a finite abelian group. The full subcategory of elementary groups in $L$ will be denoted by $E$. Then we have the following:

THEOREM 1. $\rho|E: \eta| E \rightarrow \chi^{2} \mid E$ is an isomorphism.

Duality for compact groups in $L$. We define a morphism $f \epsilon$ $\operatorname{Hom}(G, H)$ to be proper if $f$ is open considered as a function from $G$ to its image in $H$. (Thus a "proper morphism" as the term is used here may not be a "proper map" as the term is used in general topology.)

Proposition 1. If $f: G \rightarrow H$ is proper and $f(G)$ is open in $H$, then $f^{*}$ is proper.

Proof. Let $M$ be a compact neighborhood of 0 in $H$ and $W$ a closed neighborhood of 0 in $T$ which contains no proper subgroups of T. $(M, W)$ denotes the set of all characters $\alpha$ in $H^{*}$ for which $\alpha(M) \subset W$. The set of $(M, W)$ obtained from all possible such choices of $M$ and $W$ forms a base of compact neighborhoods of 0 in $H^{*}$. Suppose now that $M$ is chosen to be a compact neighborhood of 0 in $f(G)$. Since $f$ is proper and $G$ is locally compact, we may find a compact neighborhood $N$ of 0 in $G$ such that $f(N)=M$. Then $(N, W)$ is a neighborhood of 0 in $G^{*}$ and $f^{*}(M, W)=(N, W) \cap f^{*}\left(H^{*}\right)$ is a compact neighborhood of 0 in $f^{*}\left(H^{*}\right)$. The properness of $f^{*}$ follows from the open mapping theorem [2, Theorem 5.29, p. 42] applied to the open subgroup of $H^{*}$ generated by $(M, W)$.

Proposition 2. $\chi$ takes a short proper exact sequence $0 \rightarrow K \stackrel{i}{\rightarrow}$ $G \stackrel{j}{\rightarrow} H \rightarrow 0$ to a sequence $0 \leftarrow K^{*} \stackrel{i^{*}}{\leftarrow} G^{*} \stackrel{j^{*}}{\leftarrow} H^{*} \leftarrow 0$ in which $j^{*}$ is proper and exactness holds at $G^{*}$ and $H^{*}$. If in addition $K$ is an open subgroup of $G$, then the sequence induced by $\chi$ is also proper exact.

Proof. It is now easy to see that $H^{*}$ is isomorphic to the closed subgroup of $G^{*}$ consisting of those characters of $G$ which are trivial on $K$, via the proper injection $j^{*}$. The kernel of $i^{*}$ is also the set of characters on $G$ which are trivial on $K$. This proves exactness at $G^{*}$ and $H^{*}$. Now, because $T$ is a divisible group, any character on $H$ extends to a homomorphism (not necessarily continuous) from $G$ to $T$. If $H$ is an open subgroup, then any such extension will be continuous on $G$, so the proper map $i^{*}$ will be a surjection.

Using the $(M, W)$ notation of the proof of Proposition 1, we see that if $G$ is discrete and $M=\{0\}$ in $G$, then $(M, W)=G^{*}$, so $G^{*}$ is 
compact. On the other hand, if $G$ is compact and $M=G$, then $(M, W)=\{0\}$, so $G^{*}$ is discrete. We now let $A$ be the subcategory of discrete groups in $L$ and $A_{0}$ the subcategory of discrete elementary groups (isomorphic to $\left.Z^{j} \oplus F\right) . C$ is the subcategory of compact groups and $C_{0}$ the subcategory of compact elementary groups (isomorphic to $\left.T^{i} \oplus F\right)$ in $L$.

We can make a directed set $I$ into a category by declaring, for $i, j \in I$, that Hom $(i, j)$ consist of exactly one element if $i \leqq j$ and be empty otherwise. Then, for our purposes, a direct system in $A_{0}$ is a covariant functor $U$ from a directed set to $A_{0}$. We shall often write $U_{i}$ for $U(i)$ and $u_{i j}$ for $U(\operatorname{Hom}(i, j))$. We let $D A_{0}$ be the collection of direct systems in $A_{0}$ whose morphisms are all injective. $D A_{0}$ becomes a category when we define a morphism from $U: I \rightarrow A_{0}$ to $V: J \rightarrow A_{0}$ to be a pair $(m, \varphi)$, where $m: I \rightarrow J$ is a functor (orderpreserving map) and $\varphi$ is a natural transformation from $U$ to $V \circ m$.

Well-known properties of abelian groups include the fact that each element $U$ of $D A_{0}$ has a direct $\operatorname{limit} \lim U\left(\right.$ or $\left.\lim U_{i}\right)$ which will be an object in $D$. In fact, a necessary and sufficient condition for a discrete group $G$ to be isomorphic to $\lim U$ is the existence of injective morphisms $g_{i}: U_{i} \rightarrow G$, one for each $i$, such that $g_{j} \circ u_{i j}=g_{i}$ whenever $i \leqq j$, and the union of the images $g_{i}\left(U_{i}\right)$ is all of $G$. The result is that lim: $D A_{0} \rightarrow A$ can be regarded as a covariant functor, since if $(m, \varphi)$ is a functor from $U$ to $V$ in $D A_{0}$, the universal property of $\lim U$ guarantees existence of a unique morphism $\lim (m, \varphi): \lim U \rightarrow$ $\underset{\rightarrow}{\lim } \vec{V}$ making the following diagram commutative for every $\overrightarrow{i \in I} \overrightarrow{\text { : }}$

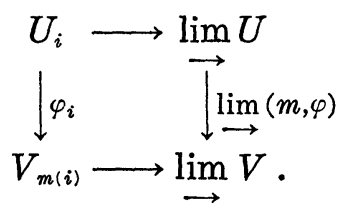

Similarly, an inverse system in $C_{0}$ is a contravariant functor $U$ from a directed set to $C_{0}$. The category of all inverse systems in $C_{0}$ all of whose morphisms are surjective is denoted by $I C_{0}$. A morphism from $U: I \rightarrow C_{0}$ to $V: J \rightarrow C_{0}$ in $I C_{0}$ is a pair $(m, \varphi)$ where $m: J \rightarrow I$ is a functor and $\varphi: U \circ m \rightarrow V$ is a natural transformation. Also, any inverse system in $I C_{0}$ has a projective $\operatorname{limit} \lim U$ which will be an object of $C$. Further, any object $G$ of $C$ is isomorphic to $\lim U$ if and only if there exists a surjective morphism $g_{i}: G \rightarrow U_{i}$ for each $i$ such that $u_{i j} \circ g_{j}=g_{i}$ whenever $i \leqq j$ and the intersection of the kernels of the $g_{i}$ is $\{0\}$ in $G$. (The usual condition [10, p. 25] is that every neighborhood of 0 in $G$ contain $\operatorname{Ker} g_{i}$ for some $i$, but if 
the intersection of the $\operatorname{Ker} g_{i}$ is $\{0\}$ and $N$ is any open neighborhood of 0 in $G$, then by compactness we can find a finite number of $g_{i}$ the intersection of whose kernels is contained in $N$. Picking an index $j$ greater than these $i$ gives us $\operatorname{Ker} g_{j} \subset N$.) Again in this situation $\lim (m, \varphi)$ for a morphism $(m, \varphi)$ in $I C_{0}$ is defined by the universal property, and $\lim$ becomes a covariant functor. We call $D A_{0}$ and $I C_{0}$ convergence structures on $A$ and $C$, respectively.

By Proposition 2, if $U \in D A_{0}$ then $\chi \circ U \in I C_{0}$. This correspondene $U \mapsto \chi \circ U$ gives us a functor which we denote by $D \chi: D A_{0} \rightarrow I C_{0}$. Similarly, $\chi$ induces the functor $I \chi: I C_{0} \rightarrow D A_{0}$.

Proposition 3. $\chi$ is continuous on $A$ with respect to $D A_{0}$; that $i s$, the two functors $\chi \circ \lim$ and $\lim \circ D \chi: D A_{0} \rightarrow C$ are naturally isomorphic.

Proof. Let $U \in D A_{0}$ and $G=\lim U_{i}$ with $g_{i}: U_{i} \rightarrow G$ the associated injections. We must show that $\overrightarrow{G^{*}} \simeq \lim \left(U_{i}^{*}\right)$. It is clear that each $g_{i}^{*}: G^{*} \rightarrow U_{i}^{*}$ is surjective. Let $0 \neq \alpha \in \overleftarrow{G}^{*}$. We shall show the existence of an index $i$ with $g_{i}^{*}(\alpha) \neq 0$. We know $\alpha(x) \neq 0$ for some $x \in G$, and then $x=g_{i}(y)$ for some $i$ and $y \in G_{i}$. Then for this $i$, $g_{i}^{*}(\alpha)(y)=\alpha\left(g_{i}(y)\right) \neq 0$. So $\left(\lim U_{i}\right)^{*} \simeq \lim \left(U_{i}^{*}\right)$. The fact that we have a natural isomorphism follows from the universal property.

Proposition $4 . \quad \chi$ is continuous on $C$ with respect to $I C_{0}$; that $i s$, the functors $\chi \circ \lim$ and $\lim \circ I \chi: I C_{0} \rightarrow A$ are naturally isomorphic.

Proof. Let $U \in I C_{0}$ and $G=\lim U_{i}$ with $g_{i}: G \rightarrow U_{i}$ the associated surjections. Clearly each $g_{i}^{*}$ is injective. To show that $G^{*}$ is isomorphic with $\lim \left(U_{i}^{*}\right)$, we must show that every $\alpha \in G^{*}$ is equal to $g_{i}^{*}(\beta)$ for some $\vec{i}$ and some $\beta \in U_{i}^{*}$. Let $W$ be a neighborhood of 0 in $T$ containing no proper subgroups of $T$. Let $M$ be a neighborhood of 0 in $G$ with $\alpha(M) \subset W$. Then we may find $U_{i}$ with $\operatorname{Ker} g_{i} \subset M$. Then $\alpha\left(\operatorname{Ker} g_{i}\right)=0$, so $\alpha$ factors; $\alpha=\beta \circ g_{i}$ for some $\beta \in U_{i}^{*}$. But $\beta \circ g_{i}=g_{i}^{*}(\beta)$, so we are done.

We remark that Proposition 3 above could be proved as in [6] by showing that $\chi$ has a left adjoint functor, which implies that $\chi$ takes direct limits to limits. However, Proposition 4 does not admit an analogous proof.

Proposition 5. The category $C_{0}$ is dense in $C$. That is, there is a functor $S: C \rightarrow I C_{0}$ such that the functor $\lim \circ S$ and the identity functor on $C$ are naturally isomorphic. 
Proof. This follows from the Peter-Weyl theorem [7, Theorem 33, p. 229], which says for our abelian case that the characters of any $G$ in $C$ separate the points of $G$. Let $G \in C$ and define $S(G) \in I C_{0}$ to be the collection of quotient groups $G / K$ of $G$ which are in $C_{0}$. We order them by $G / K \leqq G / N$ if $N \subset K$. Note that $G /(K \cap N)$ is isomorphic to a subgroup of $(G / K) \oplus(G / N)$, so we have a directed set. We can define $g_{K N}: G / N \rightarrow G / K$ to be the natural projection when $N \subset K$. For a morphism $f: G \rightarrow H$ in $C, S(f)$ is defined as follows: $H / K$ in $S(H)$ corresponds to $G / f^{-1}(K)$ in $S(G)$, and the map $G / f^{-1}(K) \rightarrow$ $H / K$ is the natural one induced by $f$. Then if $x \in G$, let $\alpha \in G^{*}$ with $\alpha(x) \neq 0$. Then $G / K$ is in $S(G)$, where $K=\operatorname{Ker} \alpha$, and $g_{K}(x) \neq 0$, where $g_{K}: G \rightarrow G / K$ is the natural map. The collection of $g_{K}$ exhibits $G$ as $\lim _{\leftarrow} S(G)$.

We are now ready to consider our two covariant functors $\eta$ and $\chi^{2}$ again.

THeorem 2. Pontryagin duality holds in $C$; that is, $\rho|C: \eta| C \rightarrow$ $\chi^{2} \mid C$ is an isomorphism.

Proof. $\rho \mid C_{0}$ is already an isomorphism. By Proposition 1.18 in [4, p. 115], $\rho \mid C_{0}$ extends uniquely to a natural transformation between $\eta \mid C$ and $\chi^{2} \mid C$. This extension must also be an isomorphism. But $\rho \mid C$ already extends $\rho \mid C_{0}$ so $\rho \mid C$ must be an isomorphism.

Duality of compactly generated groups in $L$. Let $C G$ be the full subcategory of the compactly generated groups in $L$.

Lemma. Suppose $G \in C G$ is generated by the compact neighborhood $M$ of 0 in $G$. Then there is a subgroup $K$ of $G, K \simeq Z^{n}$ for some $n$, such that $K \cap M=\{0\}$ and $G / K$ is compact.

Proof. This is Lemma 2.42 in [9, p. 41].

Proposition 6. If $G \in C G$, then $\rho_{G}$ is injective.

Proof. This is an easy consequence of the above lemma. Let $x \in G, x \neq 0$. Apply the lemma to $M \cup\{x\}$, which is also a compact neighborhood of 0 which generates $G$. The coset $x+K$ is not the identity element in the compact group $G / K$. Therefore, there is a character $\alpha$ of $G / K$ such that $\alpha(x+K) \neq 0$. Composing $\alpha$ with the natural projection $G \rightarrow G / K$ gives us a character on $G$ which is not trivial on $x$. Therefore $\rho_{G}(x) \neq 0$.

Theorem 3. Pontryagin duality holds in $C G$. 
Proof. Let $G \in C G$. Let $M$ be a compact neighborhood of 0 in $G$ and let $S \simeq Z^{n}$ be a subgroup of $G$ such that $S \cap M=\{0\}$ and $G / S$ is compact, as guaranteed by the lemma above. Let $Q=G / S$ and $p: G \rightarrow Q$ the natural map. Let $N$ be a compact symmetric neighborhood of 0 in $G$ such that $N+N+N \subset M$. Then $p$ maps $N$ homeomorphically onto $p(N)$. Since $Q$ is compact and $p$ is proper, there is a compact subgroup $Q_{1}$ of $Q$ such that $Q_{1} \subset p(N)$ and $Q / Q_{1}$ is compact elementary, by Proposition 5. Then $p^{-1}\left(Q_{1}\right)$ is a closed subgroup of $G$ contained in $S+N$. Letting $K=p^{-1}\left(Q_{1}\right) \cap N$, we have by the choice of $N$ that $K$ is a compact subgroup of $G$ satisfying $p(K)=Q_{1}$.

Now let $H=G / K$; we shall show that $H$ is an elementary group. First, $p$ gives rise to proper surjection $H \rightarrow Q / Q_{1}$ with kernel $S+K$ which is discrete in $H$ by the construction of $K$. Therefore $H$ is locally isomorphic with $Q / Q_{1}$, wich in turn is locally isomorphic with $R^{n}$ for some $n$. This means we have an isomorphism $f: B \rightarrow V$, where $B$ is an open ball about 0 in $R^{n}$ and $V$ is a neighborhood of 0 in $H$. Then we can extend $f$ to a proper surjective homorphism $g: R^{n} \rightarrow H_{1}$, whore $H_{1}$ is the open subgroup of $H$ generated by $V$, by defining $g(x)=n \cdot f(x / n)$, for $x \in R^{n}$ and $n$ large enough so that $x / n \in B$. Thus $H_{1} \simeq R^{a} \oplus T^{b}$ for some integers $a$ and $b$ (a quotient group of $R^{n}$ ). Since $H_{1}$ is a divisible open subgroup of $H$, we can obtain a morphism $H \rightarrow H_{1}$ which is the identity on $H_{1}$, so $H \simeq H_{1} \oplus H / H_{1}$. But $H / H_{1}$ is an elementary group since it is discrete and compactly generated. Therefore $H$ is also elementary. Now we examine the following commutative diagram:

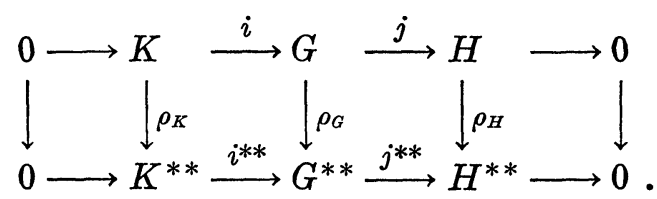

Now $K \in C$ and $H \in E$, so $\rho_{K}$ and $\rho_{H}$ are isomorphisms, while $\rho_{G}$ is injective by Proposition 6. Since $i$ is injective, we conclude that $i^{* *}$ is injective. Now briefly consider $i^{*}: G^{*} \rightarrow K^{*}$. If $i^{*}$ were not surjective, then there would be a nontrivial character on $K^{*} / i^{*}\left(G^{*}\right)$. Composing with the natural projection $K^{*} \rightarrow K^{*} / i^{*}\left(G^{*}\right)$, we get a character on $K^{*}$ which is trivial on $i^{*}\left(G^{*}\right)$. This character would then be in the kernel of $i^{* *}$, contradicting the injectivity of $i^{* *}$. Therefore, $i^{*}$ is surjective and Proposition 2 now tells us that the induced sequence

$$
0 \longleftarrow K^{*} \stackrel{i^{*}}{\longleftarrow} G^{*} \stackrel{j^{*}}{\longleftarrow} H^{*} \longleftarrow 0
$$

is a proper exact sequence, since $K^{*}$ is discrete. Thus $H^{*}$ can be regarded as an open subgroup of $G^{*}$, and so the lower sequence in 
diagram (1) is also proper exact. The 5-lemma and the open mapping theorem show that $\rho_{G}$ is an isomorphism, and we are done.

Duality for arbitrary locally compact groups. We begin with discrete groups.

Proposition 7. The category $A_{0}$ is dense in $A$. That is, there is a functor $T: A \rightarrow D A_{0}$ such that lim॰ $T$ and the identity functor on $A$ are naturally isomorphic.

Proof. Every abelian group is the direct limit of its finitely generated subgroups. The functor $T$ assigns to each $G$ in $A$ the direct system $\left(U_{i}\right)$ of finitely generated subgroups of $G$ ordered by $i \leqq j$ if $U_{i} \subset U_{j}$. A morphism $f: G \rightarrow H$ in $A$ is carried by $T$ to $T(f)$, in which each finitely generated subgroup of $G$ is mapped via the restriction of $f$ to its image in $H$.

Theorem 4. Pontryagin duality holds in $A$.

Proof. This is completely analogous to the proof of Theorem 2.

TheOREM 5. The Pontryagin duality theorem. $\rho$ is a natural epuivalence.

Proof. Let $G \in L$. Let $M$ be a compact neighborhood of 0 in $G$ and let $K$ be the subgroup of $G$ generated by $M$. Let $H=G / K$. Since $K$ is open, the induced sequence

$$
0 \longleftarrow K^{*} \longleftarrow G^{*} \longleftarrow H^{*} \longleftarrow 0
$$

is proper exact by Proposition 2. Consider diagram (1) for this $K, G$, and $H$. We have exactness at $K^{* *}$ and $G^{* *}$ in the bottom row. $H$ is discrete since $K$ is open, so $\rho_{H}$ is an isomorphism, and so $j^{* *}$ is surjective. Both rows of the diagram are proper exact and $\rho_{K}$ is also an isomorphism. Again the 5-lemma applies and $\rho_{G}$ is algebraically an isomorphism. But $\rho_{G}$ restricted to the open subgroup $K$ is the isomorphism $\rho_{K}$, so $\rho_{G}$ is also an isomorphism.

Had we extended the concepts of convergence to include direct and projective limits of nondiscrete and noncompact groups, respectively, and had we modified Propositions 3 and 4 and their proofs accordingly, we could have proved Theorem 5 differently by showing that $C G$ is dense in $L$ and $\chi$ is continuous on $L$ with respect to the modifind convergence. Theorem 3 may be treated similarly; in fact, the proof of Theorem 3 already shows that $E$ is dense in $C G$. 


\section{REFERENCES}

1. H. Cartan and R. Godement, Théorie de la dualité et analyse harmonique dans les groupes abélians localement compacts, Ann. Sci École Norm. Sup., (3) 64 (1947), 79-99. 2. E. Hewitt and K. A. Ross, Abstract Harmonic Analysis, Volume I, Academic Press, New York and Springer-Verlag, Berlin, 1963.

3. H. Heyer, Daulität Lokalkompakter Gruppen, Lecture Notes in Mathematics, Volume 150, Springer-Verlag, New York, 1970.

4. K. H. Hofmann, Categories with convergence, exponential functors, and the cohomology of compact abelian groups, Math Z., 104 (1968), 106-140.

5. E. R. van Kampen, Locally bicompact abelian groups and their character groups, Ann. of Math., (2) 36 (1935), 448-463.

6. J. W. Negrepontis, Duality in analysis from the point of view of triples, J. Algebra, 19 (1971), 228-253.

7. L. S. Pontryagin, Topological Groups, Second Edition, Gordon and Breach, New York, 1966.

8. D. W. Roeder, Functorial characterizations of Pontryagin duality, Trans. AmerMath. Soc., 154 (1971), 151-175.

9. W. Rudin, Fourier Analysis on Groups, Interscience, New York, 1962.

10. A. Weil, L'intégration dans les groupes topologiques et ses applications, Hermann, Paris, 1941 and 1951.

Received August 22, 1972 and in revised form January 26, 1973. This research was supported in part by the College Science Improvement Program of the National Science Foundation (NSF grant GY-5700).

COLORADO COLLEGE 



\section{PACIFIC JOURNAL OF MATHEMATICS}

EDITORS

RICHARD ARens (Managing Editor)

University of California

Los Angeles, California 90024

\section{R. A. Beaumont}

University of Washington

Seattle. Washington 98105

\section{J. DugundjI}

Department of Mathematics University of Southern California Los Angeles, California 90007

D. Gilbarg and J. Milgram

Stanford University

Stanford. California 94305

\section{ASSOCIATE EDITORS}
E. F. BECKENBACH
B. H. NEUMANN
F. WOLF
K. Yoshida

\section{SUPPORTING INSTITUTIONS}

\author{
UNIVERSITY OF BRITISH COLUMBIA \\ CALIFORNIA INSTITUTE OF TECHNOLOGY \\ UNIVERSITY OF CALIFORNIA \\ MONTANA STATE UNIVERSITY \\ UNIVERSITY OF NEVADA \\ NEW MEXICO STATE UNIVERSITY \\ OREGON STATE UNIVERSITY \\ UNIVERSITY OF OREGON \\ OSAKA UNIVERSITY
}

\author{
UNIVERSITY OF SOUTHERN CALIFORNIA \\ STANFORD UNIVERSITY \\ UNIVERSITY OF TOKYO \\ UNIVERSITY OF UTAH \\ WASHINGTON STATE UNIVERSITY \\ UNIVERSITY OF WASHINGTON

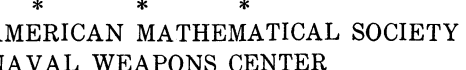

The Supporting Institutions listed above contribute to the cost of publication of this Journal, but they are not owners or publishers and have no responsibility for its content or policies.

Mathematical papers intended for publication in the Pacific Journal of Mathematics should be in typed form or offset-reproduced, (not dittoed), double spaced with large margins. Underline Greek letters in red, German in green, and script in blue. The first paragraph or two must be capable of being used separately as a synopsis of the entire paper. Items of the bibliography should not be cited there unless absolutely necessary, in which case they must be identified by author and Journal, rather than by item number. Manuscripts, in duplicate if possible, may be sent to any one of the four editors. Please classify according to the scheme of Math. Rev. Index to Vol. 39. All other communications to the editors should be addressed to the managing editor, or Elaine Barth, University of California, Los Angeles, California, 90024.

100 reprints are provided free for each article, only if page charges have been substantially paid Additional copies may be obtained at cost in multiples of 50 .

The Pacific of Journal Mathematics is issued monthly as of January 1966. Regular subscription rate: $\$ 72.00$ a year (6 Vols., 12 issues). Special rate: $\$ 36.00$ a year to individual members of supporting institutions.

Subscriptions, orders for back numbers, and changes of address should be sent to Pacific Journal of Mathematics, 103 Highland Boulevard, Berkeley, California, 94708.

\section{PUBLISHED BY PACIFIC JOURNAL OF MATHEMATICS, A NON-PROFIT CORPORATION}

Printed at Kokusai Bunken Insatsusha (International Academic Printing Co., Ltd.), 270, 3-chome Totsuka-cho. Shinjuku-ku, Tokyo 160. Japan.

Copyright (C) 1973 by Pacific Journal of Mathematics Manufactured and first issued in Japan 


\section{Pacific Journal of Mathematics}

\section{Vol. 52, No. $2 \quad$ February, 1974}

Harm Bart, Spectral properties of locally holomorphic vector-valued functions .....

J. Adrian (John) Bondy and Robert Louis Hemminger, Reconstructing infinite

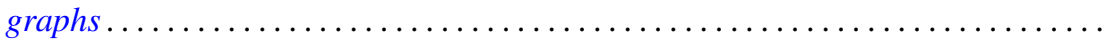

Bryan Edmund Cain and Richard J. Tondra, Biholomorphic approximation of planar

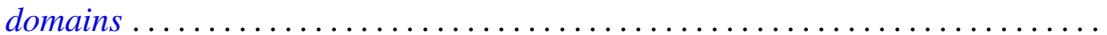

Richard Carey and Joel David Pincus, Eigenvalues of seminormal operators,

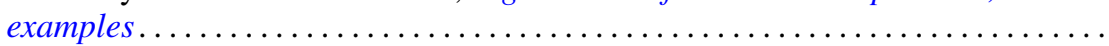

Tyrone Duncan, Absolute continuity for abstract Wiener spaces . . . . . . . . . . . . Joe Wayne Fisher and Louis Halle Rowen, An embedding of semiprime

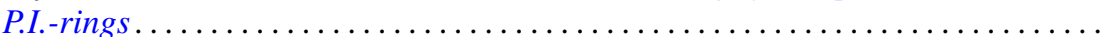

Andrew S. Geue, Precompact and collectively semi-precompact sets of semi-precompact continuous linear operators. . . . . . . . . . . . . . . .

Charles Lemuel Hagopian, Locally homeomorphic $\lambda$ connected plane continua ..... . Darald Joe Hartfiel, A study of convex sets of stochastic matrices induced by

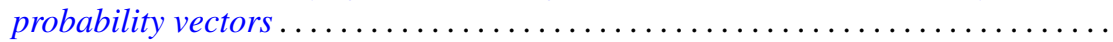

Yasunori Ishibashi, Some remarks on high order derivations $\ldots \ldots \ldots \ldots \ldots \ldots \ldots$ Donald Gordon James, Orthogonal groups of dyadic unimodular quadratic forms.

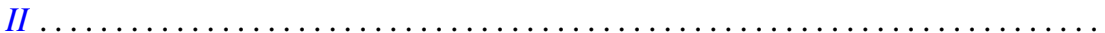

Geoffrey Thomas Jones, Projective pseudo-complemented semilattices . . . . . . . . . Darrell Conley Kent, Kelly Denis McKennon, G. Richardson and M. Schroder,

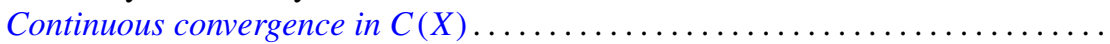

J. J. Koliha, Some convergence theorems in Banach algebras ...

Tsang Hai Kuo, Projections in the spaces of bounded linear oper

George Berry Leeman, Jr., A local estimate for typically real functions . .

475

Andrew Guy Markoe, A characterization of normal analytic spaces by the

homological codimension of the structure sheaf .........

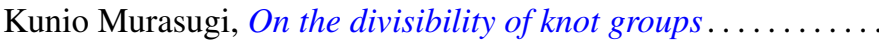

John Phillips, Perturbations of type I von Neumann algebras.

Billy E. Rhoades, Commutants of some quasi-Hausdorff matrices . .

David W. Roeder, Category theory applied to Pontryagin duality

Maxwell Alexander Rosenlicht, The nonminimality of the differential closure .

Peter Michael Rosenthal, On an inversion theorem for the general Mehler-Fock transform pair.

Alan Saleski, Stopping times for Bernoulli automorphisms

John Herman Scheuneman, Fundamental groups of compact complete locally affine complex surfaces. II. ........................

Vashishtha Narayan Singh, Reproducing kernels and operators with a cyclic vector. I. .

Peggy Strait, On the maximum and minimum of partial sums of random variables.

J. L. Brenner, Maximal ideals in the near ring of polynomials modulo 2 .

Ernst Gabor Straus, Remark on the preceding paper: "Ideals in near rings of polynomials over a field" ..........................

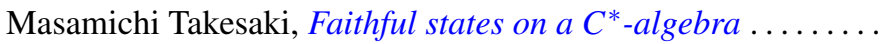

R. Michael Tanner, Some content maximizing properties of the regular simplex.

Andrew Bao-hwa Wang, An analogue of the Paley-Wiener theorem for certain

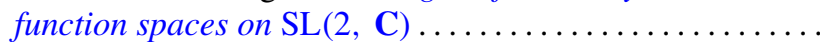

